\title{
Dependencia emocional, depresión y ansiedad en mujeres víctimas de violencia
}

Emotional dependence, depression and anxiety in women victims of violence

Dependência emocional, depressão e ansiedade nas mulheres vitimas de violência

\author{
GALIA SUSANA LESCANO LÓPEZ ${ }^{1}$ \\ VERONICA DEL ROSILLO SALAZAR ROLDAN²
}

\begin{abstract}
RESUMEN
Esta investigación tuvo por objetivo demostrar que en las mujeres víctimas de violencia, existe un nivel de relación significativa entre tres trastornos psicopatológicos: dependencia emocional, depresión y trastornos de ansiedad. Como método, se ha utilizado el procesamiento de variables a través de ecuaciones estructurales (análisis bivariado), obtenidas de la aplicación del Inventario de Dependencia Emocional - IDE, Inventario de Depresión de Beck-II (BDI-II) e Inventario de Ansiedad de Beck (BAI), en víctimas cuyos casos fueron reportados en el Instituto de Medicina Legal de Nuevo Chimbote (2019), Ancash. Como resultado, se observaron pruebas de escalas sólidas y fiables que muestran un nexo positivo entre las constantes: dependencia emocional, depresión y ansiedad, advirtiéndose una correlación de $0.58(\mathrm{p}=0.000<0.05)$, entre dependencia emocional - depresión y de 0.86 $(p=0.000<0.05)$, entre dependencia emocional - ansiedad.
\end{abstract}

Palabras clave: dependencia emocional; depresión; ansiedad; violencia.

\begin{abstract}
This research aimed to demonstrate that in women victims of violence, there is a level of significant relationship between three psychopathological disorders: emotional dependence, depression and anxiety disorders. As a method, it has been used the processing of variables through structural equations (bivariate analysis), obtained from the application of the Emotional Dependence Inventory - EDI, Beck Depression Inventory (BDI-II) and Beck Anxiety Inventory (BAI), in victims whose cases were reported in the Legal Medicine Institute of Nuevo Chimbote (2019), Ancash. As a result, it was observed evidence of solid and reliable scales that show a positive link between the constants: emotional dependence, depression and anxiety, noting a correlation of $0.58(\mathrm{p}=0.000<0.05)$, between emotional dependence - depression and $0.86(\mathrm{p}=0.000<0.05)$, between emotional dependence - anxiety.
\end{abstract}

Keywords: emotional dependence; depression; anxiety; violence. 


\section{RESUMO}

O objectivo desta investigação era mostrar que nas mulheres vítimas de violência, existe um nível significativo de relacionamento entre três transtornos psicopatológicos: dependência emocional, depressão e transtornos de ansiedade. Como método, utilizámos o processamento de variáveis através de equações estruturais (análise bivariada), obtidas a partir da aplicação do Inventário de Dependência Emocional - IDE, Inventário da Depressão Beck-II (IDB-II) e Inventário de Ansiedade Beck (IAB), em vítimas cujos casos foram relatados no Instituto de Medicina Legal de Nuevo Chimbote (2019), Ancash. Como resultado, observámos evidências de escalas sólidas e fiáveis mostrando uma ligação positiva entre as constantes: dependência emocional, depressão e ansiedade, notando uma correlação de 0,58 $(p=0,000<0,05)$, entre dependência emocional - depressão e $0,86(p=0,000<0,05)$, entre dependência emocional - ansiedade.

Palavras-chave: dependência emocional; depressão; ansiedade; violência

\section{INTRODUCCIÓN}

Dependencia emocional, depresión y ansiedad son variables psicológicas que actualmente se abordan cuando se habla de mujeres víctimas de violencia; por ello, es imprescindible estudiar la relación que existe entre estas y dicho estado. En ese sentido, los resultados obtenidos en el presente estudio permitirán tener claridad sobre los ejes que deben dirigir el enfoque de las políticas públicas que pretendan implementarse sobre el sector $\mathrm{y}$, de la misma forma, generará la posibilidad de abordar estratégicamente cada caso reportado, tratando -de forma integral-, a las víctimas.

Para este artículo, nos hemos enfocado específicamente en los actos de violencia contra las mujeres $(\mathrm{VcM})$, que se producen dentro del marco de una relación de pareja; entendiéndose este último término como una vinculación afectiva en cualquiera de sus formas de expresión (cónyuge, novio, enamorado, etc.). Su encauzamiento, basado en una perspectiva de género que reconoce a la $\mathrm{VcM}$, como resultado de las relaciones inequitativas de poder entre hombres y mujeres, construidas y naturalizadas históricamente, a través de procesos socioculturales (Vara Horna et al, 2017). En tal sentido, es relevante identificar que las desigualdades del género están asociadas con este tipo de violencia $\mathrm{y}$, de no considerarlo, se fracasará en los intentos de desarrollar estrategias que aborden esta amenaza a la salud pública (Reed et al., 2010).

También, hemos partido de la definición de violencia, esbozada en la legislación nacional peruana, la misma que hace referencia a toda acción o conducta que cause muerte, daño, sufrimiento físico, sexual o psicológico contra las mujeres, por su condición de tales, en el ámbito público o privado (Ley 30364, 2015).

De otro lado, es esencial comprender el contexto nacional. En esa línea, el reporte de la Encuesta Demográfica y de Salud Familiar (ENDES) 2019 - (Instituto Nacional de Estadística e Informática, 2020), evidencia que el 57.7\% de mujeres, fue víctima de violencia perpetrada por su pareja. Muestra además, que el 52.8\% padeció violencia psicológica o verbal (insultos, burlas, desprecio, injurias, calumnias, gritos, situaciones de control, humillaciones, 
amenazas y otros hechos que minimizan la autoestima), el 29.5\% sufrió violencia física (patadas, golpes, empujones, abofeteadas, entre otros) y el 7.1\% sufrió violencia sexual (ser obligada a tener actos sexuales o ser víctima de coacción a fin de que se ejecuten actos de esta índole que no se aprueban) (Instituto Nacional de Estadística e Informática, 2020).

Si apelamos a un ejemplo, solo la circunscripción territorial de Ancash, reportó 7750 casos atendidos en los Centros de Emergencia Mujer (CEM), durante el 2019, según la estadística reportada por el Ministerio de la Mujer y Poblaciones Vulnerables (Programa Nacional para la Prevención y Erradicación de la Violencia contra las Mujeres Integrantes del Grupo Familiar - Aurora, 2019).

En el contexto local, esta situación no parece ser ajena; tal es el caso de la provincia de Santa, escenario que se escogió para la presente investigación. El puesto de salud de la referida localidad mostró que el $66.1 \%$ de las mujeres habían sufrido violencia psicológica y el $31.4 \%$, la había padecido de forma física (Centro de emergencia, 2016). Igualmente, se evidenció que, durante el año 2019, se reportaron un total de 1255 denuncias, cuyas víctimas tenían entre 18 y 38 años.

Como efecto colateral de la incidencia en casos de VcM, es menester resaltar que, en este escenario, no solo se produce un daño psicológico, físico y emocional; sino que, además, influye en el rendimiento laboral. Así, en un estudio realizado en los docentes de la región Callao, se mostró que el $49.6 \%$ de las profesoras habían sido agredidas por sus parejas o exparejas; situación que propiciaba un desempeño docente deficiente. Como consecuencia, se podía llegar a perder hasta 165 mil horas pedagógicas anuales en las instituciones educativas públicas, lo que conllevaba a una disminución económica de US\$ 671.608 dólares al año; cifra que, en macro impacto, podía llegar a alcanzar más de US\$ 36 millones de dólares, representado el $0.47 \%$ del presupuesto nacional en educación (Vara Horna et al., 2017).

Para efectuar un análisis asertivo al respecto, es necesario preguntarnos cuáles podrían ser las posibles características socioculturales, cognitivas o conductuales que están detrás de las cifras reportadas; en tanto, la comprensión de las distintas variables que puedan identificarse permitirá ampliar el conjunto de factores que deben observarse en el momento de abordar la atención de los casos particulares. En tal sentido, es conveniente preguntarnos ¿Cómo piensa o siente una mujer mientras se encuentra en una dinámica en la que es violentada por su pareja? ¿Por qué en muchos casos la víctima sigue en el mismo escenario cíclico sin interrumpir una situación que per se, resulta lesiva? ¿Qué factores podrían influir para generar una distorsión en la percepción que las limite a ejecutar acciones que coadyuven a la preservación de su propia existencia? Por ende, ¿qué características psicopatológicas están presentes en este perfil?

El ENDES de 2019, reveló una cifra impactante que impulsó la necesidad de estudiar el perfil individual que presentan las víctimas. Frente a la pregunta ¿Por qué no buscaron ayuda? (respecto al escenario violento), un 44.8\% creyó que no era necesario, un 15.9\% precisó que sentía vergüenza y el 16.5\% mencionó tener algún tipo de miedo (temor a una nueva agresión a ella o a sus hijos, miedo a causar problemas a la persona que la violentó y miedo al divorcio o separación) (Instituto Nacional de Estadística e Informática, 2020). 
No se debe perder de vista que existen factores que predisponen las percepciones, interpretaciones y conductas de la mujer agredida, a través de formas comunes de respuesta a la frustración, que vienen condicionados por la cultura de violencia en que se encuentran, las que la hacen propensa a soportar, aceptar todo tipo de violencia considerándole, un mal inevitable (Miljánovich et al., 2013).

La tendencia actual permite advertir una multicausalidad que podría propiciar y mantener la VcM (Aiquipa, 2015); por lo que, tendremos que observar las consecuencias (como perfil individual resultante) para generar hipótesis respecto de las causas. Es así que, de manera general y no limitativa, podríamos mencionar algunas características que podrían estar presentes en las víctimas de violencia: a) poseen creencias arraigadas y actitudes tradicionales en relación al compromiso moral que tiene frente al matrimonio; b) creen que no tienen el control sobre acontecimientos de sus vidas; c) existe búsqueda constante de otra persona que calme la necesidad de afecto; d) baja autoestima; e) la presencia del sentimiento de culpa por la violencia sufrida justificando al agresor, etc. (Rhatigan \& Axsom, 2006).

En ese discurrir lógico, identificaremos los factores de riesgo que integran esta situación disfuncional. Los mismos, podrían ubicarse en diferentes niveles de análisis $\mathrm{y}$, desde la perspectiva de (Butchart et al., 2011), cuando se sitúan a escala de la sociedad, se manifestarán en los comportamientos comunitarios, interrelaciónales e individuales.

En relación con lo expuesto, hemos ahondado sobre tres variables psicopatológicas, las cuales, bajo nuestro criterio, abarcan las principales características de las víctimas de VcM y se encuadran en la esfera personalísima de la mismas: dependencia emocional, depresión y ansiedad.

La dependencia emocional, vista desde el modelo teórico de la vinculación afectiva de Castelló, será aquel patrón de conducta que implique aspectos cognitivos, motivacionales, emocionales y comportamentales conducentes a la persona que sienten que les otorga satisfacción y seguridad personal. Todo ello trae opiniones erradas acerca del amor, de la vida en pareja y de sí mismo (Aiquipa, 2015). También, ese patrón se manifestará de forma persistente e inflexible y comienza al aproximarse la adolescencia o inicios de la edad adulta (Castelló, 2005).

Este tipo de variable, como conducta límite desproporcionada, generará mayor vulnerabilidad a trastornos de distinta índole (Lemos Hoyos et al., 2012); toda vez que el sujeto dependiente se encuentra limitado para visualizarse como individuo capaz de sobrevivir lejos del objeto de su adicción. Por consiguiente, existirá un ocultamiento constante del yo (en sus innumerables expresiones); en tanto primará la visibilidad del sujeto en donde reposa la proyección de su apego. Es decir, concurrirá un autosacrificio constante, de manera voluntaria, con el objetivo permanente de sostener el vínculo $\mathrm{y}$, en dicha conducta, se experimentará satisfacción.

Es importante resaltar que los dependientes emocionales para emparejarse, se dirigen a personas con ciertas características. Por ejemplo, buscarán personas que se muestren seguras de sí mismas, egocéntricas, poco afectuosas y dominantes. Generalmente opuestos en cuanto a autoestima y valoración de sí mismos. Si la dependencia, como trastorno, es grave, con tal de no romper la relación de pareja, el dependiente aceptará menosprecio, burlas, 
humillaciones, agresiones, infidelidades continuas, entre otros; puesto que, en toda circunstancia, prevalecerán los pensamientos obsesivos y un miedo frenético a ser abandonado (Castelló, 2020).

En otras palabras, los individuos que presentan este trastorno tratan de unirse a alguna figura más fuerte que les proporcione recursos para la supervivencia y felicidad. Ya que se ven indefensos, necesitados, débiles e inútiles; por tanto, (Beck et al., 2015); forma que perciben como única para lograr la autoafirmación. En caso se coloque en riesgo la continuidad del vínculo, el individuo experimentará ansiedad, por el miedo constante a la pérdida, así como depresión. Por esta razón, muchas mujeres víctimas de violencia persisten en continuar relaciones de pareja con sus victimarios, puesto que no pueden verse lejos de los mismos por una incipiente capacidad para controlar sus impulsos.

En función de lo planteado, la dependencia emocional y la depresión son variables intrínsecamente relacionadas y presentes en la VcM; puesto que se genera un ciclo repetitivo constante, durante el sometimiento al maltrato. De allí que, en la mayor parte de los casos reportados, las mujeres no se separan de aquella situación que únicamente les trae dolor, sufrimiento y consecuencias desfavorables en su aspecto físico, emocional y de interacción. Ello evidencia una relación significativa entre dependencia emocional y la tendencia a sufrir violencia de todo tipo (Aiquipa, 2015; Momeñe et al., 2017). Del mismo modo, es innegable la repercusión psicológica generada por la exposición a actos violentos; razón por la que se percibe la presencia (causal o consecuente) de síntomas de ansiedad y depresión; somáticos según el vínculo afectivo y el tiempo de exposición a la violencia (Amor \& Echeburúa, 2010).

Dentro de ese marco, la depresión que está presente en las víctimas se caracterizará por una sensación persistente de tristeza, una tristeza diferente a otras experiencias tristes, que presenta la experiencia de pérdida de control y gran dificultad, o incluso, imposibilidad para motivarse por otros asuntos que no sean el centro de su tristeza (Moral Jimenez et al., 2018). Desde el análisis de Beck, la depresión tendrá origen en esquemas disfuncionales mantenidos, no explícitos y abstractos que regulan la información sobre sí mismo; los cuales se encuentran en la memoria a largo plazo como paradigmas superpuestos y distorsionados que no se sujetan a análisis racionales o procesos volitivos meditados. Se vinculará con pensamientos negativos sobre sí, el mundo y el futuro; lo cual podría generar desesperanza (Beck et al., 2010).

De las ideas precedentes se advierte que, tanto la dependencia emocional como la depresión, son conductas psicopatológicas que presentan esquemas cognitivos disfuncionales, así como un procesamiento de la información diferente, evidenciando distorsiones cognitivas generando un malestar emocional a quien lo padecen, lo que influye, finalmente, en su respectivo desenvolvimiento personal y social (Beck et al., 2010). Ambas conductas están estrechamente vinculadas toda vez que, ofrecerle absoluta prioridad a la pareja, viene acompañado de miedo e intolerancia a la soledad (Castillo, 2017).

De otro lado, el temor que se originará en un escenario donde predomina la violencia de parte del cónyuge, enamorado, novio u otro (de quien se depende); será un factor reactivo que provocará inevitables trastornos de ansiedad. De allí que (Villegas \& Sánchez, 2013) evidenciaron características psicológicas como: la expresión límite (percepción de la rup- 
tura como algo inconcebible y trágico), miedo a la soledad, ansiedad por separación, así como la modificación de planes (esto, para retener al perfil congruente con su esquema disfuncional). En las manifestaciones prácticas, encontraremos el miedo a la ruptura del vínculo, el miedo a ser agredida física, psicológica o sexualmente, así como el miedo de una sobreviniente agresión a los hijos o familiares cercanos.

En tal sentido, Bowlby (2017) definirá la ansiedad como la anticipación tensa e inquietante de un evento amenazante pero vago; un sentimiento de inquieto suspenso. Es un efecto negativo estrechamente relacionado con el miedo. Además, puede presentar una combinación de tensión y anticipación desagradable (Morales, 2020). Cabe precisar que los síntomas característicos de los trastornos de ansiedad se diferencian de los sentimientos habituales de nerviosismo e inquietud, ya que se presentan de manera desproporcionada, escapando del control voluntario de la persona, e interfiriendo significativa y negativamente en la vida de la persona en múltiples niveles (Puchol, 2003).

Como resultado, en el estudio de (Lara Caba et al., 2019) se advirtió que las mujeres víctimas de $\mathrm{VcM}$, presentaron síntomas de depresión leve, moderada y severa; cuyos rasgos psicopatológicos incluían trastornos de ansiedad. Así también, se debe mencionar que los trastornos de ansiedad tienden a ocurrir, de mayor manera, con trastornos depresivos; siendo que ambos, en conjunto, presentan una respuesta más pobre a los tratamientos, mayores índices de recaídas y recurrencia, así como mayor necesidad de acudir a los centros de salud (Clark \& Beck, 2010).

Por ello, resultó clara la importancia de analizar la correlación existente entre las tres variables, en tanto ha quedado exteriorizado de forma cualitativa que dichos trastornos psicopatológicos se encuentran inmersos dentro del perfil de las mujeres víctimas de violencia.

\section{METODOLOGÍA}

Se empleó el enfoque descriptivo relacional aplicándose escalas ampliamente utilizadas y validadas en diversos contextos como el Inventario para Determinar la Dependencia Emocional de Aiquipa (Aiquipa T., 2012), el Inventario para la Depresión de Beck-II (BDI-II) (Beck et al., 1996) y el Inventario de Ansiedad de Beck (BAI) (Beck et al., 1988b). La muestra de estudio estuvo conformada por 350 mujeres víctimas de violencia registradas en el Instituto de Medicina Legal de Nuevo Chimbote, mujeres entre 18 y 55 años, víctimas de todo tipo de violencia por parte de su pareja. La relación entre dependencia emocional, depresión y ansiedad se determinó a través del modelo de ecuaciones estructurales (Structural Equation Model) por mínimos cuadrados parciales (Partial Least Squares, SEM-PLS), lo que posibilita hacer predicciones a partir de variables latentes inferidas de indicadores observables (Chin, 1998). 


\section{RESULTADOS}

Tabla 1.

Descripción de dependencia emocional, depresión y ansiedad por edades

\begin{tabular}{|c|c|c|c|c|c|c|}
\hline \multicolumn{3}{|c|}{ Dependencia emocional } & \multicolumn{2}{|l|}{ Depresión } & \multicolumn{2}{|l|}{ Ansiedad } \\
\hline \multirow{4}{*}{$\begin{array}{l}18 \text { a } 29 \\
\text { años }\end{array}$} & Media & 2.47 & Media & 2.88 & Media & 2.65 \\
\hline & Desviación estándar & 0.537 & Desviación estándar & 1.194 & Desviación estándar & 0.568 \\
\hline & Asimetría & -0.252 & Asimetría & -0.591 & Asimetría & -1.360 \\
\hline & Curtosis & -1.185 & Curtosis & -1.211 & Curtosis & 0.908 \\
\hline \multirow{4}{*}{$\begin{array}{l}30 \text { a } 59 \\
\text { años }\end{array}$} & Media & 2.38 & Media & 2.83 & Media & 2.55 \\
\hline & Desviación estándar & 0.616 & Desviación estándar & 1.196 & Desviación estándar & 0.673 \\
\hline & Asimetría & -0.457 & Asimetría & -0.537 & Asimetría & -1.193 \\
\hline & Curtosis & -0.644 & Curtosis & -1.263 & Curtosis & 0.149 \\
\hline \multirow{4}{*}{$\begin{array}{l}>60 \\
\text { años }\end{array}$} & Media & 2.21 & Media & 2.79 & Media & 2.64 \\
\hline & Desviación estándar & 0.699 & Desviación estándar & 1.311 & Desviación estándar & 0.633 \\
\hline & Asimetría & -0.321 & Asimetría & -0.497 & Asimetría & -1.687 \\
\hline & Curtosis & -0.633 & Curtosis & -1.602 & Curtosis & 2.214 \\
\hline
\end{tabular}

Luego del proceso de la información de los datos obtenidos sobre dependencia emocional, depresión y ansiedad por edades, se observó que, donde la media con mayor puntuación en la dependencia emocional es el grupo de 18 a 29 años y el de menor puntuación es el grupo mayores de 60 años; en la depresión la media de mayor puntuación es en el grupo de 18 a 29 años y el de menor puntuación fue el grupo de mayores de 60 años: y en la ansiedad, la media de mayor puntuación fue el grupo de 18 a 29 años y el de menor puntuación el grupo de 30 a 59 años de edad.

En cuanto a la existencia de relación positiva y significativa entre las tres variables de estudio; se encontró que la dependencia emocional se relaciona positiva y significativamente con la depresión con un coeficiente de 0.58 lo cual indica una correlación positiva considerable y $\mathrm{p}=0.000<0.05$; la dependencia emocional se relaciona positiva y significativamente con la ansiedad con un coeficiente de correlación de 0.86 lo cual indica una correlación positiva muy fuerte y $\mathrm{p}=0.000$; $\mathrm{y}$ la depresión y ansiedad se relacionan positiva y significativamente con un coeficiente de correlación de 0.51 lo cual indica una correlación positiva considerable y $\mathrm{p}=0.000$. Tal como se ve en la tabla 2 y en la figura 1.

Tabla 2.

Correlaciones entre las variables: dependencia emocional, depresión y ansiedad

\begin{tabular}{|c|c|c|c|c|}
\hline \multicolumn{3}{|c|}{ Variables correlacionadas } & \multirow{2}{*}{$\begin{array}{l}\begin{array}{l}\text { Coeficiente de } \\
\text { correlación }\end{array} \\
.575\end{array}$} & \multirow{2}{*}{$\begin{array}{l}\mathbf{P} \\
0.000\end{array}$} \\
\hline Depresión & $<-->$ & $\begin{array}{l}\text { Dependencia } \\
\text { emocional }\end{array}$ & & \\
\hline Dependencia emocional & $<-->$ & Ansiedad & .863 & 0.000 \\
\hline Depresión & $<-->$ & Ansiedad & .514 & 0.000 \\
\hline
\end{tabular}




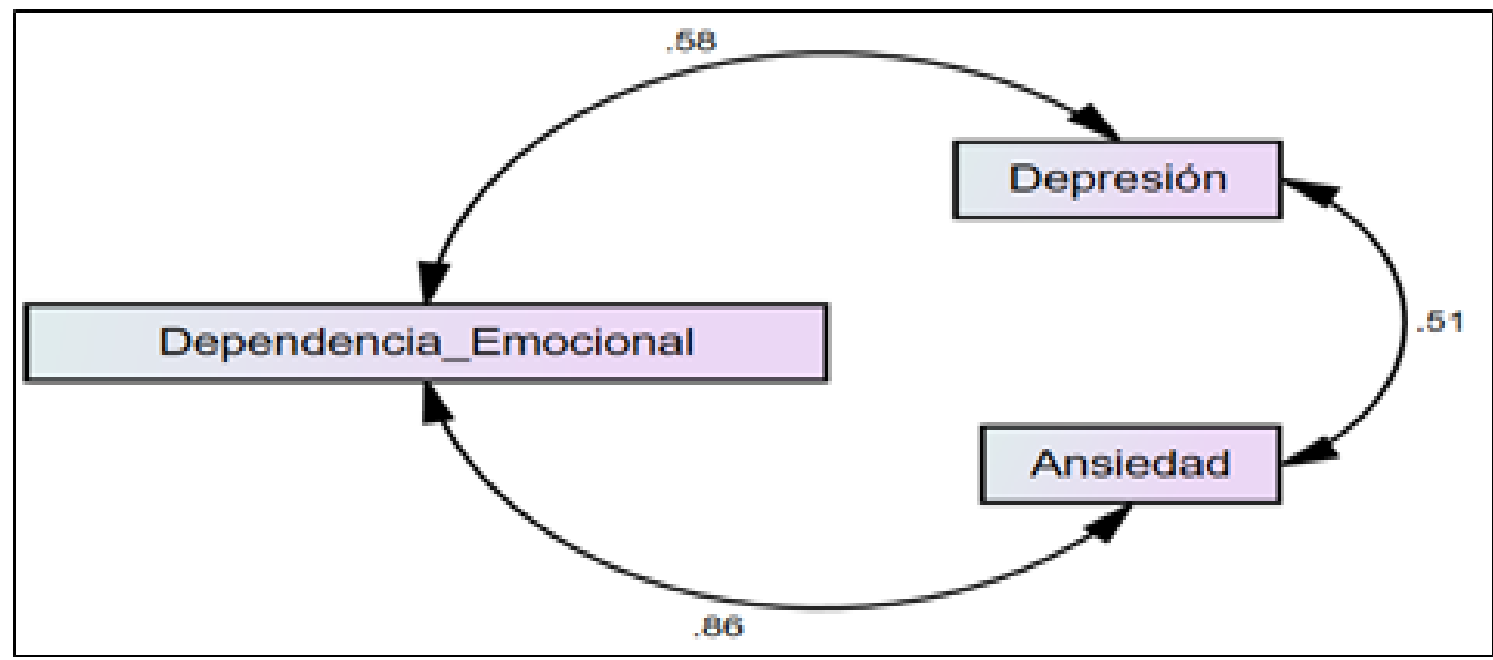

Figura 1. Correlaciones entre las variables: dependencia emocional, depresión y ansiedad Tabla 3

Correlaciones entre dependencia emocional y dimensión cognitivo afectivo de la depresión

\begin{tabular}{lllll}
\hline \hline & & & $\begin{array}{c}\text { Dependencia } \\
\text { Emocional }\end{array}$ & $\begin{array}{l}\text { Cognitivo } \\
\text { afectivo }\end{array}$ \\
\hline & \multirow{2}{*}{ Dependencia Emocional } & Coeficiente de correlación & 1.000 & $.699^{* *}$ \\
& & Sig. (bilateral) &. & .000 \\
\cline { 3 - 5 } Rho de Spearman & $\mathrm{N}$ & 350 & 350 \\
\cline { 2 - 5 } & \multirow{2}{*}{ Cognitivo afectivo } & Coeficiente de correlación & $.699^{* *}$ & 1.000 \\
& & Sig. (bilateral) & .000 & .350 \\
\hline \hline
\end{tabular}

**. La correlación es significativa en el nivel 0,01 (bilateral).

En la tabla 3, se observa que existe una relación positiva y significativa entre la dependencia emocional y la dimensión cognitivo afectivo de la depresión, con un coeficiente de correlación de 0.699 lo que indicó una correlación positiva y significativa $p=0.000<0.0$.

\section{Tabla 4}

Correlaciones entre la dependencia emocional y la dimensión somático motivacional de la depresión

\begin{tabular}{|c|c|c|c|c|}
\hline & & & $\begin{array}{l}\text { Dependencia } \\
\text { Emocional }\end{array}$ & $\begin{array}{l}\text { Somático } \\
\text { motivacional }\end{array}$ \\
\hline \multirow{4}{*}{ Rho de Spearman } & \multirow{2}{*}{$\begin{array}{l}\text { Dependencia } \\
\text { Emocional }\end{array}$} & $\begin{array}{l}\text { Coeficiente de } \\
\text { correlación }\end{array}$ & 1.000 & $.472^{* *}$ \\
\hline & & $\begin{array}{l}\text { Sig. (bilateral) } \\
\mathrm{N}\end{array}$ & 350 & $\begin{array}{l}.000 \\
350 \\
\end{array}$ \\
\hline & \multirow{2}{*}{ Somático motivacional } & $\begin{array}{l}\text { Coeficiente de } \\
\text { correlación }\end{array}$ & $.472^{* *}$ & 1.000 \\
\hline & & $\begin{array}{l}\text { Sig. (bilateral) } \\
\mathrm{N}\end{array}$ & $\begin{array}{l}.000 \\
350 \\
\end{array}$ & 350 \\
\hline
\end{tabular}

**. La correlación es significativa en el nivel 0,01 (bilateral). 
En la tabla 4, se observó que existe una relación positiva y significativa entre la dependencia emocional y la dimensión somático motivacional de la depresión, con un coeficiente de correlación de 0.472 lo cual indicó una correlación positiva media y $\mathrm{p}=0.000<0.01$.

\section{Tabla 5}

Correlaciones entre la dependencia emocional y la dimensión sensaciones motoras de la ansiedad

\begin{tabular}{lllcc}
\hline \hline & & $\begin{array}{c}\text { Dependencia } \\
\text { Emocional }\end{array}$ & $\begin{array}{c}\text { Sensaciones } \\
\text { motoras }\end{array}$ \\
\hline \multirow{5}{*}{ Rho de Spearman } & $\begin{array}{l}\text { Dependencia Emocio- } \\
\text { nal }\end{array}$ & $\begin{array}{l}\text { Coeficiente de } \\
\text { correlación }\end{array}$ & 1.000 & $.723^{* *}$ \\
& Sig. (bilateral) &. & .000 \\
\cline { 2 - 5 } & $\mathrm{N}$ & 350 & 350 \\
\cline { 2 - 5 } & \multirow{2}{*}{ Sensaciones motoras } & $\begin{array}{l}\text { Coeficiente de } \\
\text { correlación }\end{array}$ & $.723^{* *}$ & 1.000 \\
& Sig. (bilateral) & .000 &. \\
& $\mathrm{~N}$ & 350 & 350 \\
\hline \hline
\end{tabular}

**. La correlación es significativa en el nivel 0,01 (bilateral).

En la tabla 5, se observó que existe una relación positiva y significativa entre la dependencia emocional y la dimensión sensaciones motoras de la ansiedad, con un coeficiente de correlación de 0.723 lo cual indicó una correlación positiva considerable y p $=0.000<$ 0.01 .

\section{Tabla 6}

Correlaciones entre la dependencia emocional y la dimensión sensaciones cognitivas de la ansiedad

\begin{tabular}{|c|c|c|c|c|}
\hline & & & $\begin{array}{c}\text { Dependencia } \\
\text { Emocional }\end{array}$ & $\begin{array}{c}\text { Sensaciones } \\
\text { cognitivas }\end{array}$ \\
\hline \multirow{5}{*}{ Rho de Spearman } & \multirow{3}{*}{$\begin{array}{l}\text { Dependencia Emo- } \\
\text { cional }\end{array}$} & $\begin{array}{l}\text { Coeficiente de corre- } \\
\text { lación }\end{array}$ & 1.000 & $.848^{* *}$ \\
\hline & & Sig. (bilateral) & & .000 \\
\hline & & $\mathrm{N}$ & 350 & 350 \\
\hline & \multirow{2}{*}{$\begin{array}{l}\text { Sensaciones cogni- } \\
\text { tivas }\end{array}$} & $\begin{array}{l}\text { Coeficiente de corre- } \\
\text { lación }\end{array}$ & $.848^{* *}$ & 1.000 \\
\hline & & $\begin{array}{l}\text { Sig. (bilateral) } \\
\mathrm{N}\end{array}$ & $\begin{array}{l}.000 \\
350\end{array}$ & 350 \\
\hline
\end{tabular}

**. La correlación es significativa en el nivel 0,01 (bilateral).

En la tabla 6, se observa que existe una relación positiva y significativa entre la dependencia emocional y la dimensión sensaciones cognitivas de la ansiedad, con un coeficiente de correlación de 0.848 lo cual indicó una correlación positiva muy fuerte y $\mathrm{p}=0.000<0.01$. 


\section{Tabla 7}

Correlaciones entre la dependencia emocional y la dimensión somático de la ansiedad

\begin{tabular}{|c|c|c|c|c|}
\hline & & & $\begin{array}{c}\text { Dependencia } \\
\text { Emocional }\end{array}$ & Somático \\
\hline \multirow{6}{*}{$\begin{array}{l}\text { Rho de } \\
\text { Spearman }\end{array}$} & \multirow{3}{*}{$\begin{array}{l}\text { Dependencia } \\
\text { Emocional }\end{array}$} & $\begin{array}{l}\text { Coeficiente de } \\
\text { correlación }\end{array}$ & 1.000 & $.886^{* *}$ \\
\hline & & Sig. (bilateral) & . & .000 \\
\hline & & $\mathrm{N}$ & 350 & 350 \\
\hline & \multirow{3}{*}{ Somático } & $\begin{array}{l}\text { Coeficiente de } \\
\text { correlación }\end{array}$ & $.886^{* *}$ & 1.000 \\
\hline & & Sig. (bilateral) & .000 & . \\
\hline & & $\mathrm{N}$ & 350 & 350 \\
\hline
\end{tabular}

**. La correlación es significativa en el nivel 0,01 (bilateral).

En la tabla 7, se observó que existe una relación positiva y significativa entre la dependencia emocional y la dimensión somático de la ansiedad, con un coeficiente de correlación de 0.886 lo cual indicó una correlación positiva muy fuerte y $\mathrm{p}=0.000<0.01$.

\section{DISCUSIÓN}

Del análisis cualitativo y cuantitativo precedente, se advertirá que la dependencia emocional, depresión y ansiedad se manifiestan con mayor intensidad, en mujeres víctimas de violencia de 18 a 29 años, lo que coincide con el estudio de Castelló, en tanto la dependencia afectiva, empieza durante la adolescencia o a inicios de la edad adulta; trastorno que -en su expresión límite- propiciará una mayor exposición a dinámicas violentas en los vínculos de pareja.

En cuanto a la relación que se encontró utilizando el análisis bivariado de ecuaciones estructurales (para relacionar las tres variables), se determina el nivel de relación entre dependencia emocional bajo la teoría de vinculación afectiva de Castelló y depresión y ansiedad de Beck; puesto que, al ser dependiente, buscará a toda costa, proyectar su necesidad afectiva, cognitiva y conductual en un perfil que le proporcione continuidad de los esquemas disfuncionales desarrollados. En esa línea, tan minusválida y poco capaz será la percepción que el individuo afectado (en este caso, la mujer) tenga de sí mismo que perseguirá, a cualquier costo, mantener la satisfacción que le genera la cercanía al objeto de su apego desproporcionado (Beck et al., 2015). Visto desde otra arista, experimentará temor excesivo, por la sola posibilidad de la ruptura del vínculo, siendo incapaz de dominar los impulsos que la acercan al ser del que depende; aunque esto implique sufrir violencia de todo tipo (Aiquipa, 2012).

A su vez, la exposición constante a un escenario violento también generará temor por posibles agresiones sobrevinientes a sí (de carácter físico, psicológico o sexual) o a las personas de su entorno cercano (hijos o familiares). Este patrón conductual entre el victimario y la víctima, movilizarán una incesante predictibilidad sobre futuras reacciones violentas, lo que, de forma inevitable provocará la cronicidad de los trastornos ansiosos ya subyacentes en la dependencia emocional que padece. 
Lo antes expuesto, generará una constante: múltiples pensamientos negativos sobre sí, el mundo y el futuro (es decir, en el espacio individual, relacional - social y la vinculación de ambos escenarios en el tiempo); esquemas formados que posiblemente se han mantenido de forma implícita como creencias que se superponen frente a cualquier proceso racional, casi de manera automática (Beck et al., 2010). En síntesis, una conducta psicopatológica depresiva que se vinculará de manera directa con la dependencia emocional (en una aparente relación simbiótica continua).

En lo que corresponde a la relación significativa entre dependencia emocional y ansiedad, se comprueba lo que precisó Castelló Blasco, toda vez que mientras mayor sea el nivel de dependencia, mayor será el temor al abandono y, por consiguiente, absoluta disposición a evitar, bajo cualquier motivo, la ruptura (Aiquipa, 2015; Lemos Hoyos et al., 2012; Momeñé et al. 2017; Amor \& Echeburúa, 2010; Villegas \& Sánchez, 2013).

Por último, en cuanto a la relación significativa entre Depresión y Ansiedad, se verifica lo afirmado por Beck, en tanto los trastornos de ansiedad son más propensos a concurrir con trastornos de depresión (Clark \& Beck, 2010).

\section{CONCLUSIONES}

Se concluye que la dependencia emocional, depresión y ansiedad se manifiestan en mayor intensidad en mujeres víctimas de violencia de 18 a 29 años. Así también, que existe relación entre las tres variables dependencia emocional, depresión y ansiedad. De la misma forma, que existe relación significativa entre dependencia emocional y depresión con sus dimensiones cognitivo afectivo y somático motivacional. Finalmente, que existe relación significativa entre dependencia emocional y ansiedad con sus dimensiones: sensaciones motoras, sensaciones cognitivas y somático de la ansiedad.

\section{REFERENCIAS}

Aiquipa T., J. (2012). Diseño y Validación del Inventario de Dependencia Emocional- IDE. Revista IIPSI de la Facultad de Psicología de la UNSM.

Aiquipa, J. J. (2015). Dependencia emocional en víctimas de violencia de pareja. Revista de Psicología, 412- 437.

Amor, P., \& Echeburúa, E. (2010). Claves psicológicas para la permanencia de la víctima en una relación de maltrato. Clinica Contemporánea, (2) 97-104.

Beck, A., Brown, G., Epstein, N., \& Steer, R. (1988b). An inventory for measuring clinical anxiety: psychometric properties. Journal of consulting and clinical psychology.

Beck, A., Freeman, A., Davies, D., \& y otros. (2015). Terapia Cognitiva de los transtornos de la personalidad. Paidos.

Beck, A., Rush, J., Shaw, B., \& Emery, G. (2010). Terapia cognitiva de la depresión. Bilbao: Desclée de Brower.

Beck, A., Steer, R., \& Brown, G. (1996). BDI-II. Beck Depression Inventory Second Edition. San Antonio: The Psychological Corporation. 
Bowlby, J. (2017). Vínculos afectivos. Formación, desarrollo y pérdida. Madrid: Psychological Bulletin.

Butchart, A., García-Moreno, C., \& Mikton, C. (2011). Prevención de la violencia sexual y violencia infligida por la pareja contra las mujeres: qué hacer y cómo obtener evidencias. Organización Panamericana de la Salud.

Castelló, J. (2005). Dependencia emocional :Características y tratamiento. Alianza Editorial.

Castelló, J. (12 de Agosto de 2020). Dependencia emocional. Obtenido de La dependencia emocional como un transtorno de la personalidad. http://www.dependenciaemocional. org/

Castillo, E. (2017). Dependencia emociaonal, estrategias de afrontamiento al estrés y depresión en mujeres víctimas de violencia de pareja de la ciuda de Chiclayo. Revista Paian, 36-62.

Centro de emergencia, C. (2016). Plantilla de casos de atención de violencia familiar y sexual del Centro de emergencia Chimbote. Chimbote

Chin, W. (1998). The partial least squares approach for structural equation modeling. In G. A. Marcoulides (Ed.), Methodology for business and management. Modern methods for business research. Lawrence Erlbaum Associates Publishers.

Clark, D., \& Beck, A. (2010). Terapia Cognitiva para transtornos de ansiedad: Ciencia y Práctica. Biblioteca de Psicología Desclée de Brouwer.

Fernández, F. (26 de agosto de 2019). Denuncias por violencia contra la mujer ya superan las 5 mil en la región Ancash. Obtenido de Diario el Correo: https://diariocorreo.pe/ edicion/chimbote/denuncias-por-violencia-contra-la-mujer-ya-superan-las-5-milen-la-region-ancash-906671/

González, V., Santamaría, J., Merino , L., Montero, E., Fernández, D., \& Ribas, J. (2018). Dependencia emocional en mujeres: una revisión de la literatura empírica. . Psicosomática y psiquiatría, 5, 40 - 53.

Instituto Nacional de Estadística e Informática. (2020). Encuesta Demográfica y de Salud Familiar 2019. Lima.

Laca, F., \& Mejía , J. (2017). Dependencia emocional, consciencia del presente y estilos de comunicación en situaciones de conflicto con la pareja. Enseñanza e Investigación en Psicologia, 66 -75.

Lara Caba, E., Aranda Torres, C., Zapata Boluda, R., Bretones Callejas, C., \& Alarcón, R. (2019). Depresión y ansiedad en mujeres víctimas de violencia en la relación de pareja. Revista Srgentina de Ciencias del Comportamiento, 1- 8.

Lemos Hoyos, M., Jaller Jaramillo, C., González Calle, A., Diaz León, Z., \& De la Ossa, D. (2012). Perfil cognitivo de la dependencia emocional en estudiantes universitarios de Medellín, Colombia. Universitas Psycologica, 396.

Ley 30364. (2015). "Ley para prevenir, sancionar y erradicar la violencia contra las mujeres y los integrantes del grupo familiar”. Lima: Congreso de la República. 
Miljánovich, M., Huerta, R., Campos, E., Torres, S., Vasquez, V., Vera, K., \& Díaz, G. (2013). Violencia familiar: modelos explicativos del proceso a través del estudio de casos. Revisa de Investigación en Psicología, 16,32.

Momeñe, J., Jauregui-Bilbao, P., \& Estévez - Gutierrez, A. (2017). El papel predictor del abuso psicológico y la regulación emcional en la dependencia emocional. Revista Internacional de Psicología Clínica y de la Salud, (25), 65-78.

Moral Jimenez, M., Sirvent, C., Ovejero Bernal, A., \& Cuetos, G. (2018). Emotional dependence on relationships and Artemis syndrome: explanatory mode. Terapia Pcicológia, 156-166.

Morales, J. (2020). Afrontamiento de ansiedad en mujeres víctimas de violencia de genero.

Programa Nacional para la Prevención y Erradicación de la Violencia contra las Mujeres Integrantes del Grupo Familiar - Aurora. (2019). Plantilla de casos de personas afectadas por hechos de violencia contra las mujeres integrantes del grupo familiar y/o violencia sexual. Lima.

Puchol, D. (29 de setiembre de 2003). Los trastornos de ansiedad: la epidemia silenciosa del siglo XXI. Obtenido de Psicología Clinica y Psicoterapia: https://www. psicologiacientifica.com/trastornos-de-ansiedad-epidemia/\#: :text=Los $\% 20$ S\%C3\%ADntomas $\% 20$ caracter $\%$ C3\%ADsticos $\% 20$ de $\% 2010$, del $\% 20$ control $\% 20$ voluntario $\% 20 \mathrm{de} \% 201 \mathrm{a}$

Reed, E., Raj, A., Miller, E., \& Silverman, J. (2010). Losing the "Gender" in GenderBased Violence: The Missteps of Research on Dating and Intimate Partner Violence. Violence Against Women.

Rhatigan, D., \& Axsom, D. (2006). Uso del modelo de inversión para comprender el compromiso de las mujeres maltratadas con las relaciones abusivas. Revista de violencia familiar, 153-162.

Vara Horna, A., Lescano, G., Santi, I., \& Asencios, Z. (2017). Impacto de la violencia contra las mujeres ene l desempeño laboral docente. Lima: Universidad San Martin de Porres.

Villegas, M., \& Sánchez, N. (2013). Dependencia emocional en un grupo de mujeres denunciantes de maltrato de pareja. Textos \& Sentidos, 10-29. 